\title{
Successful treatment of EGFR T790M-mutant non-small cell lung cancer with almonertinib after osimertinib-induced interstitial lung disease: a case report and literature review
}

\author{
Longqiu $\mathrm{Wu}^{1 *}$, Wenjuan Zhong ${ }^{1 *}$, An $\mathrm{Li}^{1}$, Zhengang $\mathrm{Qiu}^{1}$, Ruilian Xie ${ }^{1}$, Huaqiu Shi ${ }^{1}$, Shun $\mathrm{Lu}^{2}$ \\ ${ }^{1}$ Department of Oncology, First Affiliated Hospital of Gannan Medical University, Jiangxi, China; ${ }^{2}$ Department of Shanghai Lung Cancer Center, \\ Shanghai Chest Hospital, Shanghai Jiao Tong University, Shanghai, China \\ "These authors contributed equally to this work \\ Correspondence to: Shun Lu. Department of Shanghai Lung Cancer Center, Shanghai Chest Hospital, Shanghai Jiao Tong University, West Huaihai \\ Road 241, Shanghai 200030, China. Email: shunlu@sjtu.edu.cn.
}

\begin{abstract}
Third-generation epidermal growth factor receptor (EGFR)-tyrosine kinase inhibitors (TKIs) have revolutionized the standard treatment for EGFR T790M-positive non-small cell lung cancer (NSCLC). Osimertinib is one of the third-generation EGFR-TKIs and is currently the most advanced in clinical development. Interstitial lung disease (ILD) is a potentially fatal side effect of osimertinib use. Successful rechallenge with the second-generation TKI afatinib following osimertinib-induced ILD has been reported. However, few reports have discussed the safety and efficacy of third-generation TKI rechallenge in this patient population. In this paper, a case of lung adenocarcinoma is retrospectively analyzed, and the relevant literature is reviewed. The patient was initially diagnosed with early lung cancer, for which surgical treatment was performed. The postoperative diagnosis indicated stage IB (pT2N0M0) right lung adenocarcinoma. Genetic testing (amplification-refractory mutation system) revealed EGFR exon 19 deletion. More than 2 years after surgery, multiple metastases occurred in both lungs, so gefitinib (250 mg per day) was administered. However, 6 months after the start of gefitinib treatment, the tumor progressed. Lung tumor biopsy was performed for genetic testing (NGS) and an EGFR T790M mutation was observed. Subsequently, second-line treatment with osimertinib ( $80 \mathrm{mg}$ per day) was given for 3 months. The evaluated response suggested a partial response (PR) with the occurrence of grade 3 ILD. Pemetrexed plus bevacizumab chemotherapy was subsequently administered, resulting in stable disease. However, following a severe drug reaction after six courses, the patient's chemotherapy was discontinued. Another thirdgeneration TKI, almonertinib (110 mg per day), was rechallenged based on no ILD having been reported in a phase I/II study of this drug. After 4 months of almonertinib administration and 6 months without ILD recurrence, partial remission was attained. This is the first report of successful treatment with almonertinib after osimertinib-induced ILD. The results suggested that almonertinib had a significant effect in patients with EGFR T790M mutation, with fewer side effects and better survival benefits for patients with advanced lung cancer.
\end{abstract}

Keywords: Almonertinib; osimertinib; epidermal growth factor receptor T790M mutation (EGFR T790M mutation); non-small cell lung cancer (NSCLC); case report

Submitted Apr 27, 2021. Accepted for publication Jun 09, 2021.

doi: 10.21037/atm-21-2823

View this article at: http://dx.doi.org/10.21037/atm-21-2823 


\section{Introduction}

In recent years, the incidence and mortality of lung cancer have increased globally. In 2018, there were 18.1 million new lung cancer diagnoses and 9.6 million deaths, placing lung cancer first among malignant tumors (1). Non-small cell lung cancer (NSCLC) is the most common histological type of lung cancer, accounting for up to $85 \%$ of cases (2). The epidermal growth factor receptor (EGFR) is one of the most common driver genes in NSCLC. Compared with those in Western countries, NSCLC patients in China have a high EGFR mutation rate; notably, in females and non-smokers, the EGFR sensitive mutation rate stands at $40-50 \%(3-5)$.

Patients with advanced EGFR-mutant NSCLC are extremely sensitive to first- and second-generation EGFRtyrosine kinase inhibitors (TKIs) such as gefitinib, icotinib, erlotinib, afatinib, and dacomitinib. TKIs inhibit tumor cell growth, proliferation, and metastasis, mainly by blocking the cell signal transduction pathways. However, more than half of patients develop resistance to TKIs after 9 to 14 months of administration, and the main mechanism of acquired resistance is the presence of the EGFR T790M mutation (6-10).

Third-generation EGFR-TKIs, such as osimertinib, can inhibit acquired resistance caused by first- and secondgeneration TKIs $(11,12)$. However, osimertinib can bring a variety of drug-induced toxicities which are occasionally fatal, including interstitial lung disease (ILD). Few case studies of the situation after the occurrence of osimertinibinduced ILD have been reported, and there is no standard therapy. Herein, we report a case in which a patient with advanced EGFR T790M-positive lung adenocarcinoma developed grade 3 ILD after osimertinib treatment and was successfully treated after switching to almonertinib, a novel third-generation EGFR-TKI. We also explore the clinical efficacy and side effects of almonertinib in a literature review. We present the following article in accordance with the CARE reporting checklist (available at http://dx.doi. org/10.21037/atm-21-2823).

\section{Case presentation}

A 76-year-old female patient was admitted to the First Affiliated Hospital of Gannan Medical University on June 13, 2016 with a 1-month history of a mass in the right lung, observed through physical examination. The patient had no history of smoking or drinking, and no family history of tumors.

After admission, laboratory tests showed: neuron-specific enolase: $26.15 \mathrm{ng} / \mathrm{mL}$ (normal range, $0-12.5 \mathrm{ng} / \mathrm{mL}$ ); normal carcinoembryonic antigen (CEA) and CA125; and normal blood routine, urine routine, stool routine, liver and kidney function, electrolyte, and coagulation analysis results. Chest CT showed the lesion in the right middle lobe $(3.4 \times 4.3 \mathrm{~cm})$, with no enlarged lymph nodes observed in the interstitial space (Figure 1A). Peripheral lung cancer was considered. A head and upper abdominal CT scan showed mild brain atrophy and a descending duodenal diverticulum.

On June 16, 2016, the patient underwent thoracoscopic right middle lobectomy plus regional lymphadenectomy under general anesthesia. Postoperative pathology and immunohistochemistry revealed moderately differentiated adenocarcinoma of the right lung. Cytokeratin 7 and napsin A were positive; Ki-67 was $10 \%$ positive; and CK5/6, CD56, CgA, Syn, and p63 were all negative. According to the $8^{\text {th }}$ edition of the American Joint Committee on Cancer (AJCC) TNM staging system, the patient was diagnosed with stage IB right lung adenocarcinoma (pT2N0M0). The amplification-refractory mutation system indicated EGFR exon 19 deletion. After the operation, the patient was subject to regular follow-up.

In January 2019, the patient developed a recurrent cough and occasional chest tightness. CT examination revealed multiple nodules in both lungs (Figure 1B). Tumor progression was considered, so gefitinib (Iressa, $250 \mathrm{mg}$ per day) was given orally. After administration for 3 and 5 months, the patient attained a PR (Figure 1C) according to RECIST 1.1, and the main adverse event (AE) was grade 1 diarrhea. However, disease progression was observed on August 6, 2019 (Figure 1D). Reexamination by CT showed bilateral pulmonary nodule enlargement. Firstgeneration EGFR-TKI drug resistance was considered to have occurred. On August 13, 2019, the patient underwent a CT-guided percutaneous lung biopsy. Pathology and immunohistochemistry revealed lung adenocarcinoma. CK, thyroid transcription factor-1 (TTF-1), and napsin A were all positive; Ki-67 was $10 \%$ positive; and ALK (D5F3) was negative. Subsequent next-generation sequencing indicated EGFR T790M mutation, suggesting the patient's sensitivity to third-generation EGFR-TKIs. From August 22, 2019, the patient thereby received osimertinib (80 $\mathrm{mg}$ per day).

PR was achieved after osimertinib administration for 1 and 3 months (Figure 1E,F). However, after the third month, the patient developed a recurrent cough and chest 

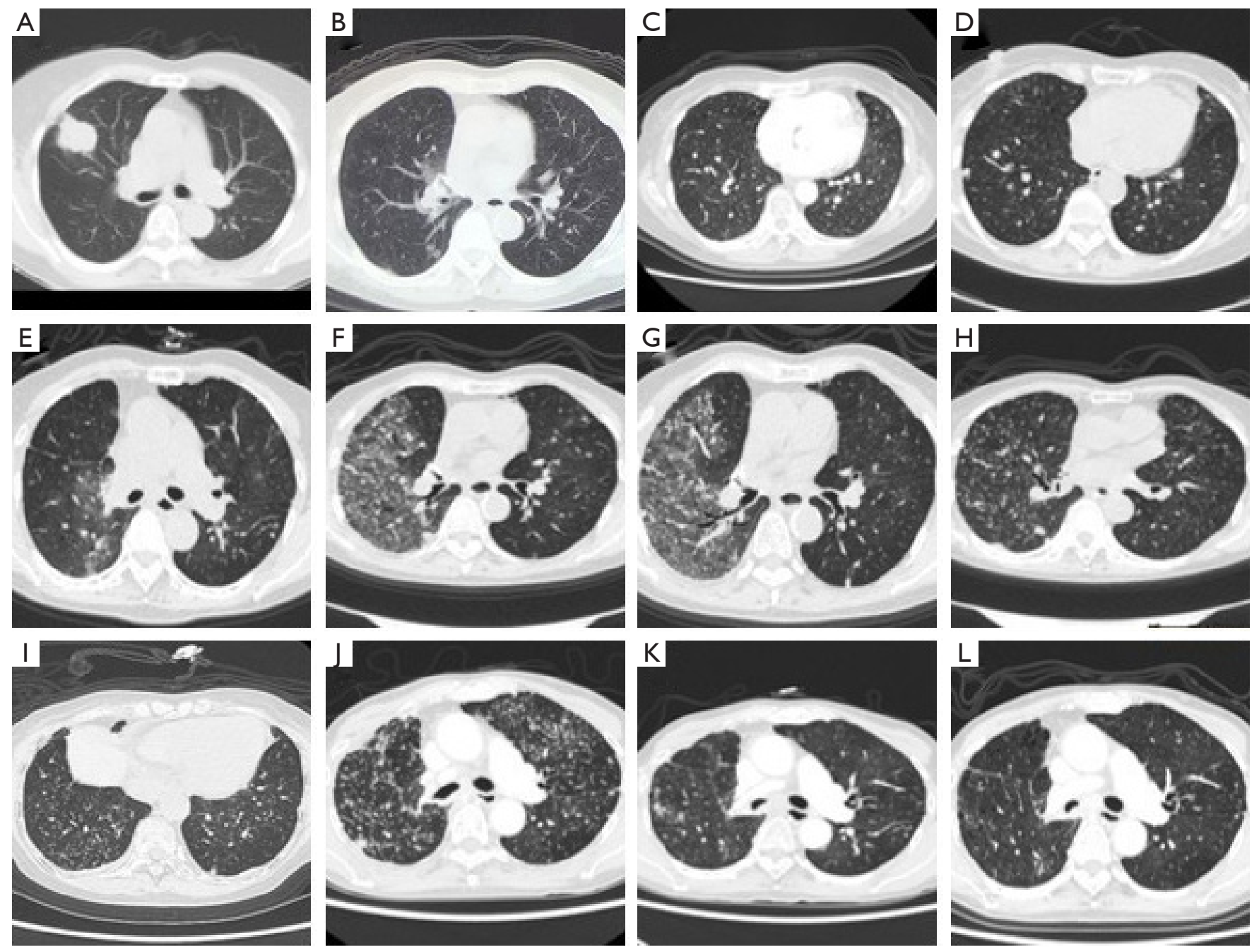

Figure 1 The clinical course according to CT scan findings. (A) Baseline CT scan at diagnosis in Jun, 2016. (B) PD after surgery in Jan, 2019. (C) PR on gefitinib in May, 2019. (D) PD on gefitinib in Aug, 2019. (E) PR on osimertinib in Sep, 2019. (F) PR on osimertinib in Nov, 2019. (G) CT revealed the ground glass opacity to be significantly reduced in Dec, 2019. (H) PD after the discontinuation of osimertinib on Jan, 2020. (I) SD on chemotherapy in Feb, 2020. (J) SD on chemotherapy in May, 2020. (K) PR on almonertinib in Sep, 2020. (L) PR on almonertinib in Nov, 2020. PD, progressive disease; PR, partial response.

pain. Chest CT on November 29, 2019, revealed no significant changes in the multiple metastases in both lungs; however, the ground-glass opacity in the right lung had progressed further, and a new lesion had formed in the left lower lobe (Figure $1 F)$. Drug-induced ILD was considered to have occurred.

To further investigate the lung lesions, electronic bronchoscopy was performed; mild inflammatory changes were observed in bilateral bronchi, and the cause of the uplift in the medial segment of the right middle lobe remained to be investigated. Under a microscope, squamous epithelial cells, ciliated columnar epithelial cells, and some macrophages were observed in the patient's bronchoalveolar lavage fluid. Based on the patient's symptoms, imaging, and bronchoalveolar lavage cytology, the oral administration of osimertinib was identified as the cause of the grade 3 ILD. Osimertinib was subsequently discontinued, and methylprednisolone (40 mg per day) combined with antitussive, antiasthmatic, analgesic, and other symptomatic treatments was given.

After 10 days, the patient's symptoms of cough and chest tightness showed significant improvement, and chest CT 
revealed the ground glass opacity to be significantly reduced (Figure 1G). Reexamination by chest CT on January 3, 2020 showed that the pulmonary infection was alleviated, but the lesions in the bilateral lungs were increased and enlarged, indicating progressive disease (Figure 1H). Four courses of pemetrexed (700 mg on day 1) plus bevacizumab (300 $\mathrm{mg}$ on day 1) were conducted on January 7, January 28, February 18, and March 10, 2020. On February 16 during the treatment period, chest CT indicated multiple metastases in both lungs, with some nodules being slightly smaller than before; response evaluation indicated stable disease (Figure 1I). On April 3 and 25, 2020, two courses of pemetrexed $(700 \mathrm{mg})$ alone were given. CT examination on May 15, 2020 still showed stable disease, as indicated by multiple metastases in both lungs without obvious changes compared with previous images (Figure 17). However, the patient stopped chemotherapy due to obvious anorexia after treatment, and was switched to oral almonertinib (110 mg per day).

The patient was evaluated as PR on September 6, 2020 and November 2, 2020. CT showed that the pulmonary nodules had shrunk (Figure $1 K, L$ ). To date, the patient has experienced no obvious adverse drug reactions to oral almonertinib and ILD did not recur. The patient's current performance status score is 1 , and no chest tightness or shortness of breath affecting light physical activity has been reported.

All procedures performed in studies involving human participants were in accordance with the ethical standards of the institutional and/or national research committee(s) and with the Helsinki Declaration (as revised in 2013). Written informed consent was obtained from the patient.

\section{Discussion}

Lung cancer treatment has entered into the era of molecular targeted therapy. Compared with traditional chemotherapy, targeted drugs have greatly improved the prognosis and prolonged the survival of NSCLC patients with positive driver genes, and AEs of grade 3 and above have been significantly reduced $(8,13-15)$.

EGFR is a tyrosine kinase (TK) receptor located in the cell membrane. EGFR can facilitate signal conduction through binding, activation, and dimerization with extracellular epidermal growth factor, as well as through phosphorylation to activate downstream signaling, thereby affecting cell proliferation (16). EGFR mutation is a common driver mutation in NSCLC, which can cause abnormal activation of TKs, resulting in a series of uncontrolled cell biological behaviors, and is only seen in tumor tissue (17). The TK region is the main gene mutation region and includes exons 18-21. A partial deletion in exon 19 (19del) and an exon 21 point mutation (21-L858R) are both sensitive mutations and account for approximately $85 \%$ of EGFR mutations (18). EGFR-TKIs can bind to the TK in the intracellular domain of EGFR and competitively inhibit TK phosphorylation, thereby blocking signaling in tumor cells and inhibiting their growth and metastasis.

Currently, first-line treatments that have been approved for patients with advanced EGFR-mutant NSCLC include the first-generation (gefitinib, erlotinib, and icotinib), second-generation (afatinib and dacomitinib), and thirdgeneration (osimertinib) EGFR-TKIs. The median progression-free survival (PFS) of patients treated with firstand second-generation TKIs is $9-14$ months $(8,9,13,14)$. In the case reported herein, the patient developed drug resistance following 6-month oral administration of the first-generation TKI gefitinib, and the PFS was relatively short. The T790M mutation, which accounts for approximately $50 \%$ of resistance mechanisms, is the most common mechanism associated with drug resistance $(19,20)$; it occurs when the $790^{\text {th }}$ amino acid in exon 20 of EGFR changes from threonine to methionine, resulting in steric hindrance and the failure of the EGFR-TKI to block the activation of EGFR.

Osimertinib is the first third-generation EGFRTKI to be approved by FDA for marketing. It is used to treat patients with EGFR T790M-positive NSCLC who experience secondary drug resistance following first- or second-generation EGFR-TKI therapy. In early clinical trials (AURA1 and AURA2), osimertinib exhibited good safety and efficacy $(11,21,22)$. Subsequently, the prospective, multicenter, open-label phase III clinical study AURA3 showed that compared with chemotherapy, osimertinib significantly increased the PFS (median: 10.1 vs. 4.4 months) and objective response rate $(71 \%$ vs. $31 \%)$ of patients with advanced T790M-positive NSCLC who acquired resistance to first- or second-generation EGFR-TKIs. Osimertinib was also found to possess a greater ability to penetrate the blood-brain barrier than TKIs of other generations $(21,23)$.

Common side effects of osimertinib include diarrhea, rash, dry skin, and neurotoxicity. Studies have also shown that ILD will develop in $3.3 \%$ of patients after around 34 days of osimertinib treatment, with $0.5 \%$ of patients who receive osimertinib progressing to a life-threatening condition $(24,25)$. Although the incidence of osimertinibinduced ILD has been reported to be as high as $12-13 \%$ 
$(26,27)$, the underpinning mechanism is still not entirely clear, and only a few related reports have been published. In a rare case report of osimertinib-induced ILD, the pathological manifestation was acute eosinophilic pneumonia (28). During CT examination, TKI-induced ILD can manifest as a single ground-glass opacity, multiple consolidations, scattered multiple ground-glass opacities with interlobular septal thickening, or extensive groundglass opacity lesions or consolidation areas in both lungs with tractive bronchiectasis (29). Lung biopsy is the only diagnostic method for ILD, but it can also result in trauma to patients and is therefore rarely used in the clinical setting. Patients who develop ILD of grade 3 or above usually stop osimertinib treatment permanently, and are given steroids as soon as possible. In the present case, the patient achieved PR after 1 month of orally administered osimertinib (80 mg per day) therapy. Also, CT showed scattered groundglass opacities in the lung. After 3 months of treatment, grade 3 ILD occurred, presenting as extensive ground-glass opacities in both lungs, and the patient was forced to stop taking osimertinib. To date, no studies have explored the clinical efficacy and safety of the substitution or alternation of osimertinib or other third-generation TKIs.

Almonertinib is a novel, third-generation EGFR-TKI used to treat patients with EGFR T790M-positive NSCLC who have progressed on or after therapy with other EGFRTKIs. A phase I dose-escalation study of almonertinib demonstrated its compelling safety and tolerability (30). The results of a phase II clinical study of almonertinib were reported at the 2019 World Conference on Lung Cancer (WCLC 2019): the overall response rate was 68.9\%; the disease control rate was $93.4 \%$; the median PFS was 12.3 months; and the median duration of response was 12.4 months. Almonertinib also exhibited good control over central nervous system (CNS) metastases: the CNS overall response rate was $60.9 \%$; CNS disease control rate was $91.3 \%$; the median CNS PFS was 10.8 months; and the median CNS duration of response was 11.3 months (31). Currently, a phase III clinical study of almonertinib as a first-line treatment for patients with advanced EGFR-positive NSCLC is in progress.

Regarding their mechanisms of action, almonertinib and osimertinib can irreversibly bind to the EGFR ATP binding site through a covalent bond, and irreversibly bind to the EGFR T790M resistance mutation. Compared with osimertinib, almonertinib has a cyclopropyl group modification in its structure, which combines with EGFR T790M perfectly, resulting in high inhibitory activity, selectivity, and excellent pharmacokinetic properties. Furthermore, the degradation products of almonertinib in vivo present a low affinity for and weak inhibitory effect on wild-type EGFR, which can reduce common side effects, such as diarrhea and rash. The lipophilicity of the cyclopropyl group also ensures good blood-brain barrier penetration, so almonertinib is more efficient effect for patients with brain metastasis of lung cancer. The main AEs caused by almonertinib include digestive system reactions, anemia, fatigue, abdominal pain, and skin reactions (pruritus and paronychia), and the overall incidence of AEs is less than $30 \%$. No cases of ILD relating to almonertinib have been reported $(30,31)$.

Several cases of EGFR-TKI rechallenge following osimertinib-induced ILD have been reported in the literature $(27,32)$. There are two ways to carry out rechallenge: (I) readministration of osimertinib with or without steroids, or (II) switching to an EGFR-TKI other than osimertinib. Considering that our patient had severe ILD, under the premise of effective osimertinib treatment, we selected almonertinib, as another third-generation TKI, as subsequent therapy, which also achieved a significant effect.

In summary, we have reported a case of successful treatment of EGFR T790M-positive NSCLC with almonertinib after osimertinib-induced ILD. This is the first reported case of switching from osimertinib to another third-generation TKI following the occurrence of osimertinib-induced ILD. So far, the patient has received almonertinib treatment for almost 7 months and has attained a sustained PR without ILD or other obvious drug side effects. Careful monitoring of any relevant symptoms of ILD may be important to prevent the development of life-threatening ILD in the follow-up course. Almonertinib benefited our patient and reduced the occurrence of AEs; it could therefore be a promising therapeutic strategy for other patients with EGFR T790M-positive NSCLC. However, whether or not there is cross-resistance to EGFR-TKIs needs to be further investigated.

\section{Acknowledgments}

We thanked the patient and her family for publication of this case report.

Funding: None.

\section{Footnote}

Reporting Checklist: The authors have completed the CARE 
reporting checklist. Available at http://dx.doi.org/10.21037/ atm-21-2823

Conflicts of Interest: All authors have completed the ICMJE uniform disclosure form (available at http://dx.doi. org/10.21037/atm-21-2823). SL received research support from AstraZeneca, BMS, Hansoh, Hengrui Therapeutics, Hutchison MediPharma and Roche. Received speaker fees from Astra Zeneca, Hansoh, Hengrui Therapeutics and Roche. Performed consulting or advisory role for Astra Zeneca, Boehringer Ingelheim, GenomiCare, Hutchison MediPharma, Menarini, Pfizer, PrIME Oncology, Roche, Simcere, Yuhan Corporation and ZaiLab. The other authors have no conflicts of interest to declare.

Ethical Statement: The authors are accountable for all aspects of the work in ensuring that questions related to the accuracy or integrity of any part of the work are appropriately investigated and resolved. All procedures performed in studies involving human participants were in accordance with the ethical standards of the institutional and/or national research committee(s) and with the Helsinki Declaration (as revised in 2013). Written informed consent was obtained from the patient.

Open Access Statement: This is an Open Access article distributed in accordance with the Creative Commons Attribution-NonCommercial-NoDerivs 4.0 International License (CC BY-NC-ND 4.0), which permits the noncommercial replication and distribution of the article with the strict proviso that no changes or edits are made and the original work is properly cited (including links to both the formal publication through the relevant DOI and the license). See: https://creativecommons.org/licenses/by-nc-nd/4.0/.

\section{References}

1. Bray F, Ferlay J, Soerjomataram I, et al. Global cancer statistics 2018: GLOBOCAN estimates of incidence and mortality worldwide for 36 cancers in 185 countries. CA Cancer J Clin 2018;68:394-424.

2. Lu S, Yu Y, Yang Y. Retrospect and Prospect for Lung Cancer in China: Clinical Advances of Immune Checkpoint Inhibitors. Oncologist 2019;24:S21-S30.

3. Shi Y, Au JS, Thongprasert S, et al. A prospective, molecular epidemiology study of EGFR mutations in Asian patients with advanced non-small-cell lung cancer of adenocarcinoma histology (PIONEER). J Thorac Oncol
2014;9:154-62.

4. Gou LY, Wu YL. Prevalence of driver mutations in nonsmall-cell lung cancers in the People's Republic of China. Lung Cancer (Auckl) 2014;5:1-9.

5. Wu YL, Zhong WZ, Li LY, et al. Epidermal growth factor receptor mutations and their correlation with gefitinib therapy in patients with non-small cell lung cancer: a meta-analysis based on updated individual patient data from six medical centers in mainland China. J Thorac Oncol 2007;2:430-9.

6. Yu HA, Arcila ME, Rekhtman N, et al. Analysis of tumor specimens at the time of acquired resistance to EGFR-TKI therapy in 155 patients with EGFR-mutant lung cancers. Clin Cancer Res 2013;19:2240-7.

7. Rosell R, Carcereny E, Gervais R, et al. Erlotinib versus standard chemotherapy as first-line treatment for European patients with advanced EGFR mutation-positive non-small-cell lung cancer (EURTAC): a multicentre, open-label, randomised phase 3 trial. Lancet Oncol 2012;13:239-46.

8. Wu YL, Zhou C, Hu CP, et al. Afatinib versus cisplatin plus gemcitabine for first-line treatment of Asian patients with advanced non-small-cell lung cancer harbouring EGFR mutations (LUX-Lung 6): an open-label, randomised phase 3 trial. Lancet Oncol 2014;15:213-22.

9. Shi YK, Wang L, Han BH, et al. First-line icotinib versus cisplatin/pemetrexed plus pemetrexed maintenance therapy for patients with advanced EGFR mutation-positive lung adenocarcinoma (CONVINCE): a phase 3, open-label, randomized study. Ann Oncol 2017;28:2443-50.

10. Fukuoka M, Wu YL, Thongprasert S, et al. Biomarker analyses and final overall survival results from a phase III, randomized, open-label, first-line study of gefitinib versus carboplatin/paclitaxel in clinically selected patients with advanced non-small-cell lung cancer in Asia (IPASS). J Clin Oncol 2011;29:2866-74.

11. Jänne PA, Yang JC, Kim DW, et al. AZD9291 in EGFR inhibitor-resistant non-small-cell lung cancer. N Engl J Med 2015;372:1689-99.

12. Yang J, Ramalingam SS, Jnne PA, et al. LBA2_ PR: Osimertinib (AZD9291) in pre-treated pts with T790M-positive advanced NSCLC: Updated phase 1 (P1) and pooled phase $2(\mathrm{P} 2)$ results. J Thorac Oncol 2016,11:S152-3.

13. Mok TS, Wu YL, Thongprasert S, et al. Gefitinib or carboplatin-paclitaxel in pulmonary adenocarcinoma. N Engl J Med 2009;361:947-57.

14. Zhou C, Wu YL, Chen G, et al. Erlotinib versus 
chemotherapy as first-line treatment for patients with advanced EGFR mutation-positive non-small-cell lung cancer (OPTIMAL, CTONG-0802): a multicentre, open-label, randomised, phase 3 study. Lancet Oncol 2011;12:735-42.

15. Wu YL, Zhou C, Liam CK, et al. First-line erlotinib versus gemcitabine/cisplatin in patients with advanced EGFR mutation-positive non-small-cell lung cancer: analyses from the phase III, randomized, open-label, ENSURE study. Ann Oncol 2015;26:1883-9.

16. Ito M, Codony-Servat C, Codony-Servat J, et al. Targeting PKC1-PAK1 signaling pathways in EGFR and KRAS mutant adenocarcinoma and lung squamous cell carcinoma. Cell Commun Signal 2019;17:137.

17. Rodrigues MA, Gamba CO, Faria JA, et al. Inner nuclear membrane localization of epidermal growth factor receptor (EGFR) in spontaneous canine model of invasive micropapillary carcinoma of the mammary gland. Pathol Res Pract 2016;212:340-4.

18. Tu HY, Ke EE, Yang JJ, et al. A comprehensive review of uncommon EGFR mutations in patients with non-small cell lung cancer. Lung Cancer 2017;114:96-102.

19. Cortot $A B$, Jänne PA. Molecular mechanisms of resistance in epidermal growth factor receptor-mutant lung adenocarcinomas. Eur Respir Rev 2014;23:356-66.

20. Matikas A, Mistriotis D, Georgoulias V, et al. Current and Future Approaches in the Management of Non-Small-Cell Lung Cancer Patients With Resistance to EGFR TKIs. Clin Lung Cancer 2015;16:252-61.

21. Mok TS, Wu YL, Ahn MJ, et al. Osimertinib or PlatinumPemetrexed in EGFR T790M-Positive Lung Cancer. N Engl J Med 2017;376:629-40.

22. Goss G, Tsai CM, Shepherd FA, et al. Osimertinib for pretreated EGFR Thr790Met-positive advanced nonsmall-cell lung cancer (AURA2): a multicentre, open-label, single-arm, phase 2 study. Lancet Oncol 2016;17:1643-52.

23. Ahn MJ, Tsai CM, Yang JCH, et al. 3083 AZD9291 activity in patients with EGFR-mutant advanced nonsmall cell lung cancer (NSCLC) and brain metastases:

Cite this article as: Wu L, Zhong W, Li A, Qiu Z, Xie R, Shi H, Lu S. Successful treatment of EGFR T790M-mutant non-small cell lung cancer with almonertinib after osimertinib-induced interstitial lung disease: a case report and literature review. Ann Transl Med 2021;9(11):950. doi: 10.21037/atm-21-2823
Data from Phase II studies. Eur J Cancer 2015;51:S625-6.

24. Komada F. Analysis of Time-to-onset of Interstitial Lung Disease after the Administration of Small Molecule Molecularly-targeted Drugs. Yakugaku Zasshi 2018;138:229-35.

25. U.S. Food and Drug Administration, Center for Drug Evaluation and Research: Application number: 208065Orig1s000. Tagrisso/osimertinib. 2015.

26. Ohe Y, Imamura F, Nogami N, et al. Osimertinib versus standard-of-care EGFR-TKI as first-line treatment for EGFRm advanced NSCLC: FLAURA Japanese subset. Jpn J Clin Oncol 2019;49:29-36.

27. Kodama H, Wakuda K, Yabe M, et al. Retrospective analysis of osimertinib re-challenge after osimertinibinduced interstitial lung disease in patients with EGFRmutant non-small cell lung carcinoma. Invest New Drugs 2021;39:571-7.

28. Tachi H, Shiozawa T, Sakai C, et al. Osimertinib-Induced Interstitial Lung Disease Presenting as Eosinophilic Pneumonia. J Thorac Oncol 2017;12:e118-20.

29. Endo M, Johkoh T, Kimura K, et al. Imaging of gefitinibrelated interstitial lung disease: multi-institutional analysis by the West Japan Thoracic Oncology Group. Lung Cancer 2006;52:135-40.

30. Yang JC, Camidge DR, Yang CT, et al. Safety, Efficacy, and Pharmacokinetics of Almonertinib (HS-10296) in Pretreated Patients With EGFR-Mutated Advanced NSCLC: A Multicenter, Open-label, Phase 1 Trial. J Thorac Oncol 2020;15:1907-18.

31. Lu S, Wang Q, Zhang G, et al. A Multicenter, Open-label, Single-arm, Phase II Study: The Third Generation EGFR Tyrosine Kinase Inhibitor Almonertinib (HS-10296) for Pretreated EGFR T790M-Positive Locally advanced or Metastatic Non-Small Cell Lung Cancer. J Thorac Oncol 2019;14:S208-9.

32. Nishima S, Miyanaga A, Saito S, et al. Successful Treatment with Afatinib after Osimertinib-induced Interstitial Lung Disease in a Patient with EGFR-mutant Non-small-cell Lung Cancer. Intern Med 2021;60:591-4. 